\title{
THE USE OF AUXETIC MATERIALS IN SMART STRUCTURES
}

\author{
E. P. HADJIGEORGIOU ${ }^{1}$ AND G. E. STAVROULAKIS ${ }^{2,3 *}$ \\ ${ }^{1}$ Department of Materials Science and Engineering, University of Ioannina, GR-45110 Ioannina, Greece \\ ${ }^{2}$ Department of Matematics, University ofIoannina, GR-45110Ioannina, Greece \\ ${ }^{3}$ Department of Civil Engineering, Technical University of Braunschweig, Germany
}

(Rec. 14 December 2004)

\begin{abstract}
This paper presents a study of the implications of using auxetic materials in the design of smart structures. By using auxetic materials as core and piezoelectric actuators as face layers to provide control forces, the problem of the shape control of sandwich beams is analyzed under loading conditions. The mechanical model is based on the shear deformable theory for beams and the linear theory of piezoelectricity. The numerical solution of the model is based on superconvergent (locking-free) finite elements for the beam theory, using Hamilton's principle. The optimal voltages of the piezo-actuators for shape control of a cantilever beams with classical and auxetic material are determined by using a genetic optimization procedure. Related numerical solutions of static problems demonstrate the role of auxetic material in the deformation, shape control and stress distribution of the beam and related twodimensional composite elastic structures.
\end{abstract}

Key words: Auxetic materials, smart beams and plates, shape control, finite element analysis, genetic optimization.

\section{INTRODUCTION}

Due to the increasing demand of high structural requirements, the modelling and control of flexible structures have received considerable interest among the research community. Recently, considerable attention has been focused on the development of advanced structures with integrated distributed control and self-monitoring capabilities. These structures are frequently classified as smart or intelligent structures (see [1]). The smart structures are primarily employed to control the static and dynamic responses of distributed parameter systems operating under variable service conditions. Piezoelectric materials used as sensor or actuator elements in smart structures. These materials respond to mechanical forces and generate an electric charge. Relatively fewer investigations have been done on the shape control resulting from bending, despite its practical importance (see [2-4]). Readjusting the shapes and the focal points of space antennas or the contours of aircrafts, space-crafts, and ship surfaces are some examples requiring structural shape control. In designing smart struc-

Corresponding author: e-mail: gestavr@cc.uoi.gr; fax: 0030-26510-98201 
tures integrated with piezoelectric actuators, engineers have to select the appropriate type of actuators, their locations on the structure, and the amount of actuation energy to be applied to the actuators. A recent review article by Irschik [5] describes relevant applications of static and dynamic shape control of structures by piezoelectric actuation. In situations where one is dealing with share deformable beams the effect of transverse shear deformation is significant and it may be necessary to use the more accurate shear deformation (Timoshenko) beam theory. In our previous work [6], a detailed shear-deformable mechanical model was formulated for a sandwich beam with face piezoelectric layers using a superconvergent finite element formulation, following the work of Friedman and Kosmatka [7], and Reddy [8]. The purpose of this paper is the application of this model to shape control of beams with classical and auxetic material. The materials with a negative Poisson's ratio are called auxetic materials. A large number of recent articles discuss their origin, methods for their numerical modeling at a structural or microstructural level and their potential applications. The reader may consult, among others, the papers [9-12] and the references given there. The purpose of this paper is to present a concrete application on a shape control problem for a beam with auxetic core and, by means of simplified numerical results, demonstrate possible aspects of using auxetic components in smart elastic structures. Extension to further multifield or dynamical problems will be the subject of a future investigation.

The shape control problem is formulated in the framework of structural optimization, as a least square optimization problem. The numerical solution within MATLAB is based on the genetic algorithm developed by Houck's et al. [13].

\section{THE MATHEMATICAL MODEL}

An elastic beam with rectangular cross section having length $L$, width $\mathrm{b}$ and thickness $h$ is considered. A pair of piezoelectric layers with thickness $h_{S}$ and $h_{A}$ is symmetrically perfectly bonded at the top and the bottom surfaces of the beam, as shown in Fig. 1.

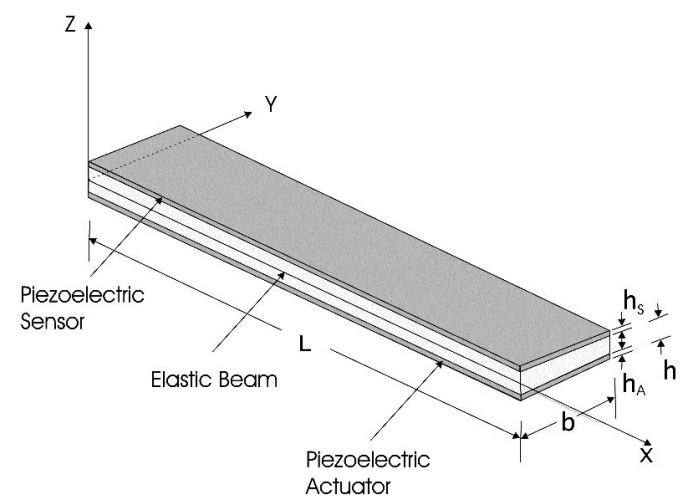

Fig. 1. A sandwich beam with face piezoelectric layers 


\subsection{Kinematics and piezoelectric equations}

A Cartesian coordinate system $(x, y, z)$ is defined on the sandwich beam, where the $x$ axis coincides with the centroidal axis of the beam and $y$ and $z$ coinside with the principal axes of the cross section. It is further assumed that the centroidal axis coincides with the elastic axis of the beam so that bending-torsion coupling is negligible. Moreover, the analysis is restricted to the behavior in the $x-z$ plane only so that the displacement relations in the $\mathrm{x}, \mathrm{y}$ and $\mathrm{z}$ directions can be written as (Timoshenko's beam theory):

$$
u_{1}(x, y, z, t) \approx z \psi(x, t), \quad u_{2}(x, y, z, t) \approx 0, u_{3}(x, y, z, t) \approx w(x, t)
$$

where $w$ is the transverse displacement of the points of the centroidal axis and $\psi$ is the rotation of the beam cross section about the positive $y$-axis. The nonzero strain components of the beam are determined by using the Eq. (1) as

$$
\varepsilon_{x}=z \frac{\partial \psi}{\partial x}, \quad \gamma_{x z}=\psi+\frac{\partial w}{\partial x}
$$

Generally, the linear piezoelectric coupling between the elastic field and the electric field with no thermal effects can be expressed by the following constitutive equations (Tiersten [14])

$$
\begin{gathered}
\{\sigma\}=[Q]\{\varepsilon\}-[e]^{T}\{E\}, \\
\{D\}=[e]\{\varepsilon\}+[\epsilon]\{E\},
\end{gathered}
$$

where $\{\sigma\}$ is the stress vector, $\{\varepsilon\}$ is the strain vector, $\{D\}$ is the electric displacement vector, $\{E\}$ is the electric field vector, $\{Q\}$ is the elastic constant matrix, $[e]$ is the piezoelectric constant matrix and [ ] is the dielectric constant matrix. Equation (3) describes the converse piezoelectric effect and Eq. (4) describes the direct piezoelectric effect. The direct relationship is employed in the modelling of the sensing capabilities of the piezoelectric layer whereas actuator interactions are modelled by using the converse relationship.

By assuming that each layer of the sandwich beam possesses a plane of material symmetrically parallel to the $x-y$ plane, the constitutive Eqs. (3), (4), under the kinematical assumptions (1), can be written for each layer as

$$
\begin{gathered}
\left\{\begin{array}{l}
\sigma_{x} \\
\tau_{x z}
\end{array}\right\}=\left[\begin{array}{cc}
Q_{11} & 0 \\
0 & Q_{55}
\end{array}\right]\left\{\begin{array}{l}
\varepsilon_{x} \\
\gamma_{x z}
\end{array}\right\}-\left[\begin{array}{cc}
0 & e_{31} \\
e_{15} & 0
\end{array}\right]\left\{\begin{array}{l}
E_{x} \\
E_{z}
\end{array}\right\}, \\
\left\{\begin{array}{l}
D_{x} \\
D_{z}
\end{array}\right\}=\left[\begin{array}{cc}
0 & e_{15} \\
e_{31} & 0
\end{array}\right]\left\{\begin{array}{l}
\varepsilon_{x} \\
\gamma_{x z}
\end{array}\right\}+\left[\begin{array}{cc}
\epsilon_{11} & 0 \\
0 & \epsilon_{33}
\end{array}\right]\left\{\begin{array}{l}
E_{x} \\
E_{z}
\end{array}\right\},
\end{gathered}
$$

where the $Q_{k k}^{(i)}$ are the reduced elastic constants of $i$-th layer: 


$$
Q_{11}^{(i)}=\frac{E^{(i)}}{1-\left(v^{(i)}\right)^{2}}, \quad Q_{55}^{(i)}=G^{(i)}=\frac{E^{(i)}}{2\left(1+v^{(i)}\right)} .
$$

Furthermore, $E^{(i)}$ denotes the Young's modulus, $v^{(i)}$ is the Poisson's ratio and $G^{(i)}$ is the shear modulus of $i$-th layer.

\subsection{Equations of motion}

The equations of motion of the sandwich beam are derived from the Hamilton's principle:

$$
\delta \int_{t_{1}}^{t_{2}}\left(T-H+W_{E}\right) d t=0,
$$

where $\delta(\cdot)$ denotes the first variation operator, $T$ is the kinetic energy, $H$ is the electric enthalpy and $W_{e}$ is the work done by the external forces and moments.

The kinetic energy of the beam, by using the Eqs. (1), (2) and integrating over the total cross-section of the structure, can be written as

$$
\begin{gathered}
T=\frac{1}{2} \int_{V} \rho\{\dot{u}\}^{T}\{\dot{u}\} d V=\frac{1}{2} \int_{V} \rho\left[\left(z \dot{\psi}^{2}\right)+(\dot{w})^{2}\right] d V= \\
=\frac{1}{2} \int_{0}^{L}\left[I_{3}(\dot{\psi})^{2}+I_{1}(\dot{w})^{2}\right] d x,
\end{gathered}
$$

where

$$
\left(I_{1}, I_{3}\right)=\int_{S} \rho\left(1, z^{2}\right) d s
$$

are the linear and rotatory inertia coefficients of the total cross-section $S$ of the beam, respectively.

The electric enthalpy of the beam (see, for example, $[14,15])$, by using the Eqs. $(1,2)$ and integrating over the total cross-section $S$ of the beam, can be written as

$$
\begin{gathered}
H=\int_{V}\left(\frac{1}{2}\{\varepsilon\}^{T}[Q]\{\varepsilon\}-\{\varepsilon\}^{T}[e]^{T}\{E\}-\frac{1}{2}\{E\}^{T}[\epsilon]\{E\}\right) d V= \\
=\int_{0}^{L} \frac{1}{2}(E I)\left(\frac{\partial \psi}{\partial x}\right)^{2}+\frac{1}{2}(G A)\left(\psi+\frac{\partial w}{\partial x}\right)^{2}-M^{e l} \frac{\partial \psi}{\partial x}+ \\
-Q^{e l}\left(\psi+\frac{\partial w}{\partial x}\right)-\frac{1}{2}\left(\epsilon_{11} E_{x}^{2}+\epsilon_{33} E_{z}^{2}\right) d x
\end{gathered}
$$

with

$$
(E I)=\int_{S} z^{2} Q_{11} d s
$$




$$
\begin{gathered}
(G A)=\int_{S} k Q_{55} d s, \\
M^{e l}=\int_{S_{A}} z e_{31} E_{z} d s, \\
Q^{e l}=\int_{S_{A}} e_{15} E_{x} d s,
\end{gathered}
$$

where, $(E I)$ and $(G A)$ are the generalized elastic constants of the sandwich beam, $M^{e l}$ and $Q^{e l}$ are the electric bending moment and electric transverse shear force per unit length, respectively, both induced by the actuator face, $S_{A}$ denotes the area of the actuator's cross-section and

$$
k=\frac{10(1+v)}{12+11 v}
$$

is the shear correction factor [16].

Finally, the work of external forces is given as

$$
W_{e}=\int_{0}^{L}(q w+m \psi) d x
$$

where $q$ and $m$ are the distributed forces and moments along the length of the beam.

The two differential equations of motion and the associated boundary conditions are obtained by substituting Eqs. (8), (10) and (16) into Eq. (7) and integrating by parts, that is

$$
\begin{gathered}
\frac{\partial}{\partial x}\left[(G A)\left(\psi+\frac{\partial w}{\partial x}\right)\right]-\frac{\partial Q^{e l}}{\partial x}+q=I_{1} \frac{\partial^{2} w}{\partial t^{2}} \\
\frac{\partial}{\partial x}\left((E I) \frac{\partial \psi}{\partial x}\right)-(G A)\left(\psi+\frac{\partial w}{\partial x}\right)-\frac{\partial M^{e l}}{\partial x}+Q^{e l}+m=I_{3} \frac{\partial^{2} \psi}{\partial t^{2}}
\end{gathered}
$$

where the geometric and natural boundary conditions that must be specified at the ends of the beam $(x=0, L)$ are

Geometric

$\omega$ is specified or

$\psi$ is specified

or
Natural

$$
(G A)\left(\psi+\frac{\partial w}{\partial x}\right)-Q^{e l}=0
$$




\subsection{Finite element formulation}

Consider a beam element of length $L_{e}$ which has two mechanical degrees of freedom at each node: one translational degree of freedom $w_{1}\left(w_{2}\right)$ and one rotational degree of freedom $\psi_{1}\left(\psi_{2}\right)$, as it is shown in Fig. 2.

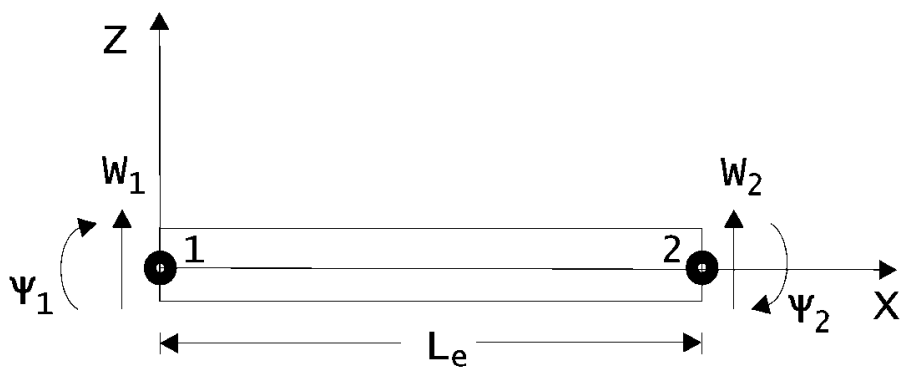

Fig. 2. Beam element

The array of nodal displacements and rotations is defined as

$$
\left\{\mathbf{X}^{e}\right\}=\left[\begin{array}{llll}
w_{1} & \psi_{1} & w_{2} & \psi_{2}
\end{array}\right]^{T}
$$

The beam element's transverse deflection and rotation are approximated by

$$
\begin{gathered}
w(x, t)=\sum_{i=1}^{4} H_{i}^{\omega}(x) X_{i}^{e}(t), \\
\psi(x, t)=\sum_{i=1}^{4} H_{i}^{\psi}(x) X_{i}^{e}(t),
\end{gathered}
$$

where $H_{i}^{\omega}$ is a cubic shape function and $H_{i}^{\psi}$ is a quadratic shape function. Thus lead to a two-node, with two degree of freedom per node, Timoshenko beam element which is impervious to shear-locking, and it is called superconvergent element [7, 8]. The dynamic matrix equation of the beam element are developed by substituting the displacement distribution (Eq. 22) into the Hamilton's principle (Eq. 7) and by carrying out the integration over the beam element length is formulated as

$$
\left[M^{e l}\right]\{\ddot{\mathbf{X}}\}^{e}+\left[K^{e}\right]\{\mathbf{X}\}^{e}=\{F\}^{e}+\left\{F_{e l}\right\}^{e}
$$

where $\left[M^{e l}\right]$ is the mass matrix, $\left[K^{e}\right]$ is the stiffness matrix, $\{F\}$ is the consistent force array and $\left\{F_{e l}\right\}$ is the electric force array.

By assembling all the elemental equations one gets the global dynamic equation

$$
[\mathbf{M}]\{\ddot{\mathbf{X}}\}+[\mathbf{K}]\{\mathbf{X}\}=\{\mathbf{F}\}+\left\{\mathbf{F}_{e l}\right\} .
$$




\section{SHAPE CONTROL AND GENETIC OPTIMIZATION PROCEDURE}

For static problems, like in the shape control studied here, the state vector $\mathbf{X}$ of the system is time-independent. Consequently, the equation of motion, Eq. (24), takes the simplified form

$$
[\mathbf{K}]\{\mathbf{X}\}=\{\mathbf{F}\}+\left\{\mathbf{F}_{e l}\right\} .
$$

With fixed, time-independent, value of the electric potential at the various actuators, one is able to control the shape of the composite smart beam. For instance, the partial or total alleviation of the deflections caused by an external loading is possible by this way. The most general problem of optimal shape control of smart beams involves the definition of the number, position and actuation voltages of the actuators such that the beam's deflections to take given values. Shape control problems can take the form of an optimization problem as follows: A given mathematical model of the system is defined and a set of input-output data for this system is available (parametrized mechanical problem), but the values of the parameters, which are involved in the system, are unknown (design or control problem). In the particular problem, each one of the actuators is considered as a separate design parameter which is the applied voltage. The minimization of the beam's deflections is the optimal design task and is equivalent to the minimization of the error function between output (mechanical response) and wished values. For the most common Euclidean norm this is a least square optimization problem. Here for simplicity, it is assumed that every actuator covers exactly the length of one finite element. A general purpose numerical algorithm for the solution of theoptimal shape design problem is the genetic optimization procedure (see [13] where the MATLAB code used in this paper is available). In the framework of the genetic optimization, the set of the unknown variables of the problem (cf. phenotype) are encoded as a chain of binary variables (cf. chromosomes). Furthermore, due to the stochastic nature of this approach, a population of test value of variables is assumed. For each set of values of the design variables, an error function is calculated. Each of this set constitutes an individual in this population. In accordance with the terminology used in genetic optimization, the minimization problem is transformed into a maximization problem for a suitably defined fitness function. The procedure is further partially inspired by Darwin's rule of the survival through natural selection. In the selection step individuals with better fitness values are given a higher probability to be mated and pass on their characteristics to the next generation. A crossover operator permits parts of the encoding string of the parents to be exchanged within the reproduction step. Finally, arbitrary parts of the information are changed at random (mutation) during the creation of the new generation. Sometimes, very good individuals are allowed to pass through the whole procedure unchanged (elitism), i.e. they are copied as they are in the next generation. For the fitness function in the particular problem studied here the most beneficial choice has been to use the natural logarithm as a scaling filter. 


\section{NUMERICAL RESULTS, RELATED OBSERVATIONS AND DISCUSSION}

\subsection{Shape control of a sandwich beam}

In what follows, the case of a cantilever sandwich beam which is schematically shown in Fig. 3, will be studied.
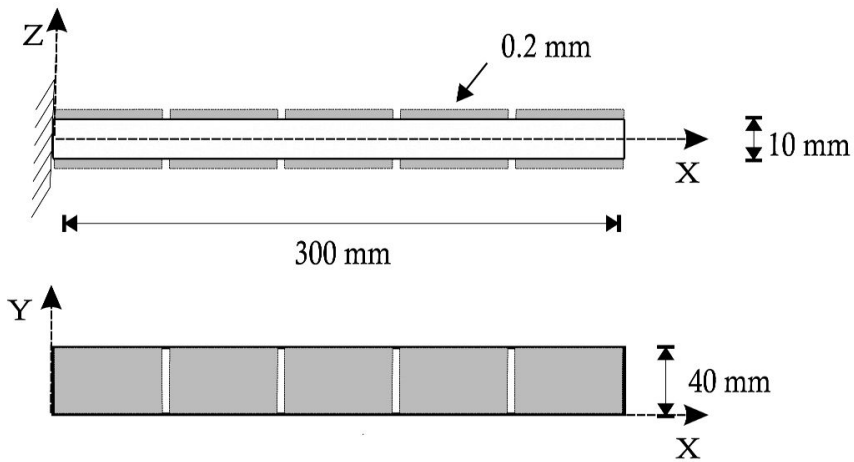

Fig. 3. A cantilever beam with surface bonded piezoelectric actuators and $x-z$ plane as bending plane

The core material is made of a graphite/epoxy composite material and the face material are PZT piezoceramic. The adhesive layers are neglected. The material properties are given in Table 1.

The beam has length equal to $300 \mathrm{~mm}$, total thickness equal to $9.6 \mathrm{~mm}$, width equal to $40 \mathrm{~mm}$, and thickness of each piezoelectric layer equal to $0.2 \mathrm{~mm}$. The sandwich beam has clamped end at the left hand side and is subjected to a fixed point loading equal to $5 \mathrm{~N}$ at the free right hand side. The beam is divided evenly into 30 finite elements. All the piezoceramics on the upper and lower surfaces of the beam are used as actuators. Equal-amplitude voltages with an opposite sign are applied to the upper and lower piezoelectric layers respectively in order to control the deformation of the beam subjected to the concentrated load. The same example has been used in our previous investigation with classical core, see [6]. In the present paper the effect of auxetic core is investigated.

Table 1. Material properties of face and core materials

\begin{tabular}{lcc}
\hline & Face & Core \\
\hline Young's moduli $(\mathrm{GPa}) E_{11}$ & 63 & 150 \\
Poisson ratio $v_{12}$ & 0.3 & 0.3 \\
Shear moduli $(\mathrm{GPa}) G_{13}$ & 24.2 & 7.1 \\
Density $\left(\mathrm{Kg} / \mathrm{m}^{3}\right) \rho$ & 7600 & 1600 \\
Piezoelectric constants $\left(\mathrm{C} / \mathrm{m}^{2}\right) e_{31}$ & 17584 & \\
Electrical permittivity $(\mathrm{F} / \mathrm{m}) \epsilon_{31}$ & $15.3 \times 10^{-9}$ & \\
Electrical permittivity $(\mathrm{F} / \mathrm{m}) \epsilon_{33}$ & $15.0 \times 10^{-9}$ & \\
\hline
\end{tabular}




\subsubsection{Consequences of auxetic materials to shape control}

In order to investigate the effect of an auxetic material on the shape control of the beam, two sandwich beams are considered: one with classical core material (having positive Poisson's ratio equal to $v=0.3$ ) and a second one with auxetic core material (having negative Poisson's ratio with a value of $v=-0.7)$. The deflection curves of these beams due to the same external loading with no activated actuators are shown in the Fig. 4. From Fig. 4 one may

Fig. 4. The centerline deflection of two cantilever sandwich beams with classical and auxetic core material

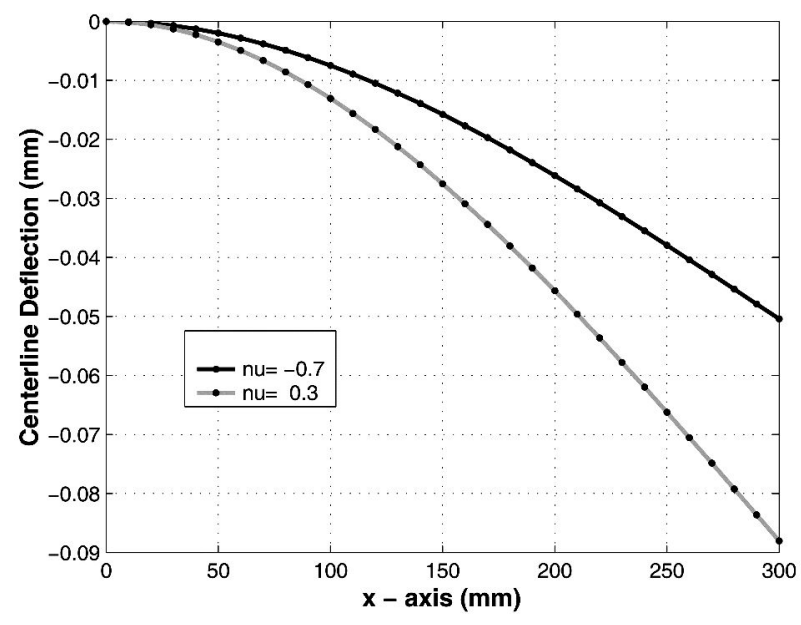

observe that the beam with auxetic core has smaller deflection than the beam with classical core material, under the same loading. When the Poisson's ratio of the core approaches the value of -1 , the structure shows a higher resistance to shear because the shear modulus $G$ of the material is with the Poisson's ratio, increase [17]. Although the structure with auxetic core material becomes stiffer, the electric voltage values of actuators for the shape control differs slightly from the corresponding values for the beam with classical core. Relative results are shown in Fig. 5.

Table 2. Shape control of a cantilever beam with optimal values of voltages for the more efficient combinations (best fitness value) of the actuator groups. Results obtained with the genetic optimization algorithm and a population size equal to 40

\begin{tabular}{lcccccc}
\hline \multirow{2}{*}{ Core Material } & \multicolumn{5}{c}{ Voltage of Actuator Group } & Fitness \\
\cline { 2 - 6 } & 1 & 2 & 3 & 4 & 5 & \\
\hline $\begin{array}{l}\text { Classical } \\
(v=0.3)\end{array}$ & 200.75 & 151.44 & 105.15 & 70.28 & 17.55 & 22.93 \\
$\begin{array}{l}\text { Auxetic } \\
(v=-0.7)\end{array}$ & 198.95 & 154.02 & 106.38 & 67.24 & 20.35 & 24.20 \\
\hline
\end{tabular}


The numerical performance of the optimal shape control algorithm has been tested with a cantilever beam modelled with 30 elements and five groups of actuators from the left side to right one. Each element is equipped with a piezoelectric actuator and each group of actuators contains six elements with equal actuation electric potential. A maximum number of 100 generations has been allowed for all examples. It should be mentioned also that an upper limit
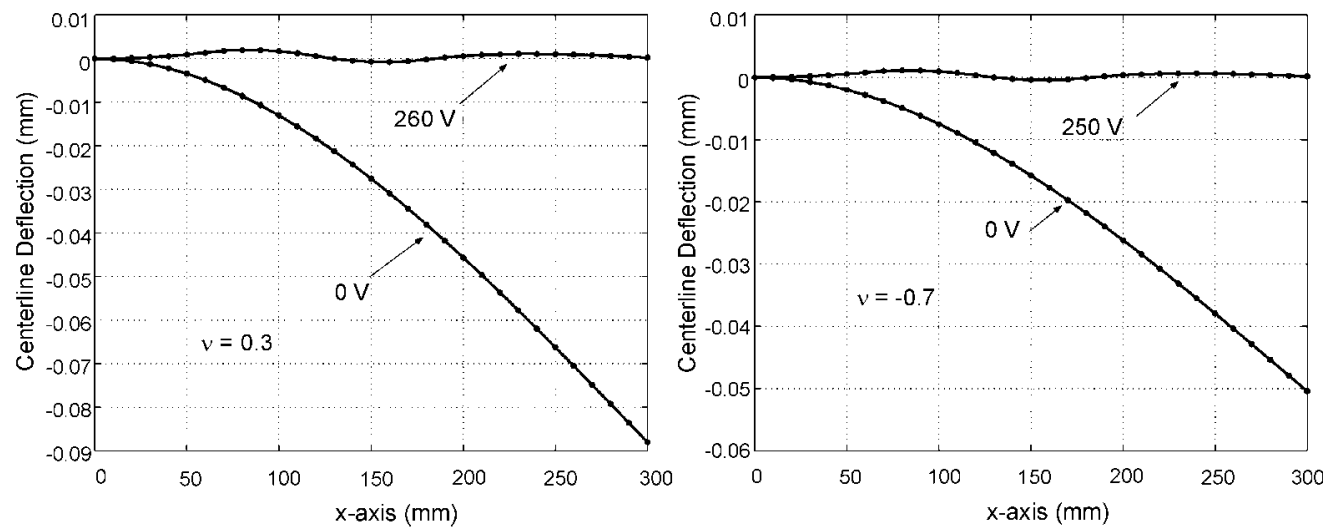

Fig. 5. The centerline deflection of the cantilever beam with two pairs of actuators located at the left end and middle span of the beam
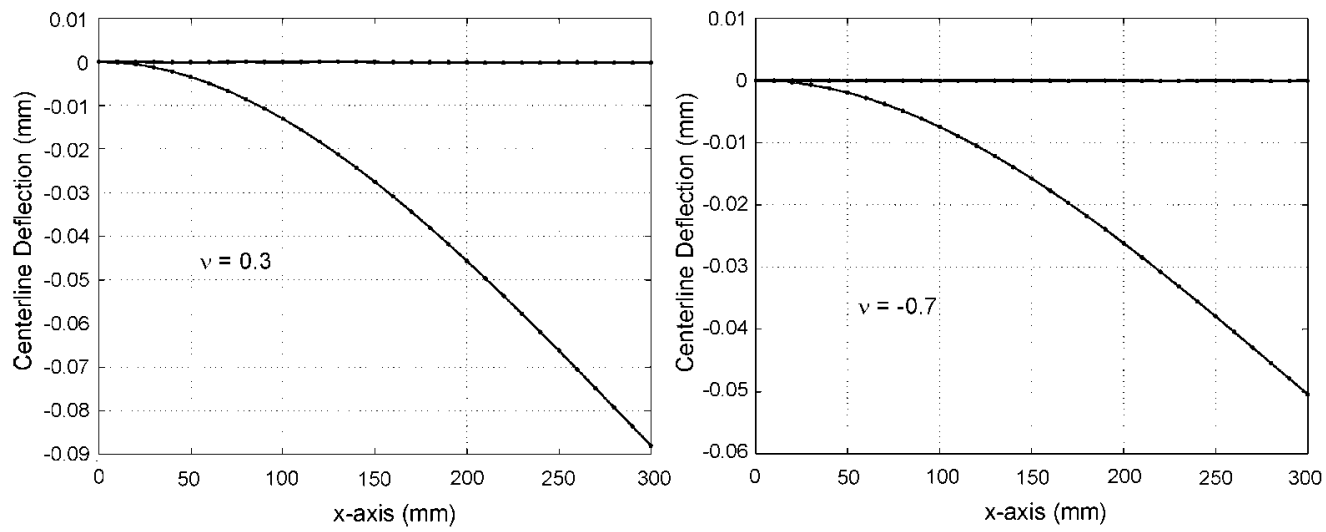

Fig. 6. The centerline of the cantilever smart beam with classical and auxetic core under the action all of the five groups of actuators with the optimal values of actuation voltages

of 500 volts has been considered for all design variables, although this limit has never been met in the numerical examples. The optimal values of voltages for the more efficient combinations of actuator groups to shape control of the beam are presented in Table 2. A graphical presentation of the these results is given in Fig. 6. 


\subsection{Effect of the auxetic material on static response}

A number of related examples demonstrate the local distribution of stresses related to auxetic material. Even in linear elasticity the auxetic effect influences certain components of the stress distribution.

\subsubsection{Distribution of stresses from actuator action}

For instance, a slight change of the von Mises stresses at the vicinity of an actuator is demonstrated in Fig. 7. This effect, which is expected to become higher for anisotropic materials, may influence quantities which are critical for the strength or the resistance of the composite to delamination or damage.

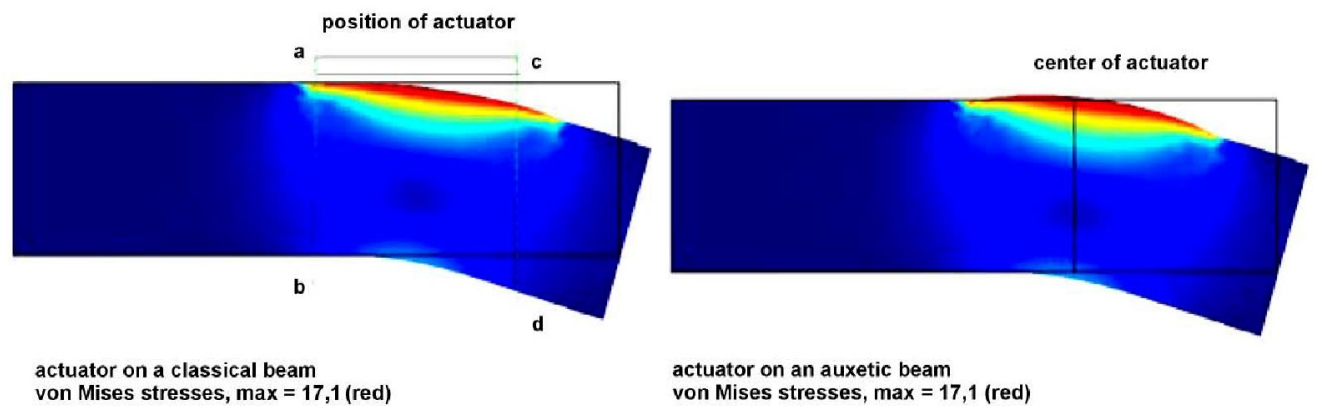

Fig. 7. Local distribution of action stresses in the core with classical and auxetic material

\subsubsection{Distribution of transverse shear stresses}

In the same spirit the shear stresses of a cantilever beam near the fixed boundary are strongly influenced from the auxetic core. The position and sign of the maximum changes, as it can be seen from Fig. 8. In order to have an accurate estimate of the shear stresses, which is

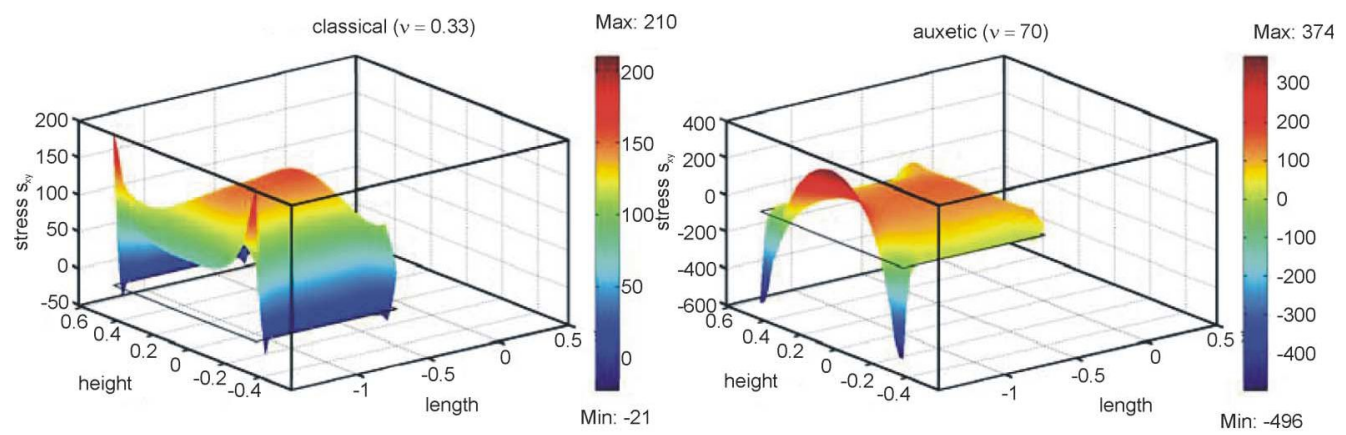

Fig. 8. Distribution of transverse shear stresses in the core of acantilever beam with classical and auxetic material 
not influenced from some simplified assumptions of a beam theory, the results have been obtained from the numerical solution of aplane stress problem with the finite element method.

\subsubsection{Shear deformable plates with classical and auxetic material}

Using the first-order shear deformation theory (Mindlin plate theory) and finite element modelling the stress distribution studied in plates with classical and auxetic materials can be studied. In this example we consider a quadrilateral plate in bending, with support boundary

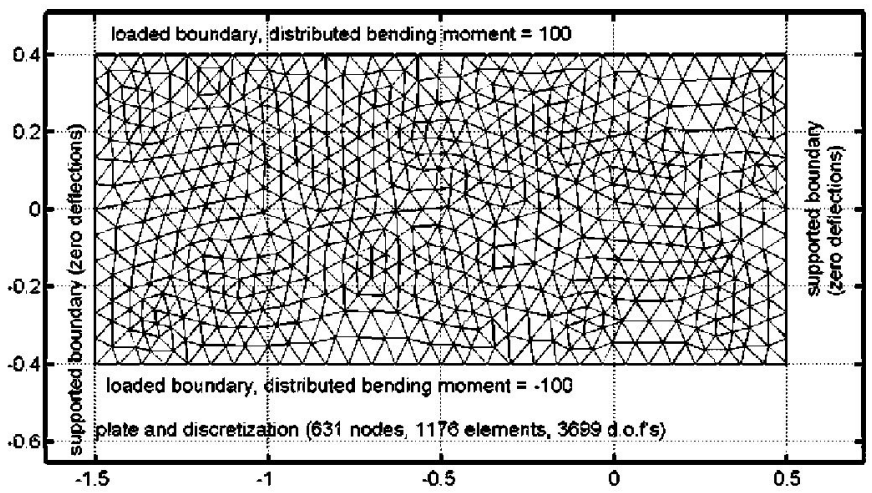

Fig. 9. Dimensions, discretization and boundary conditions of the plate in bending
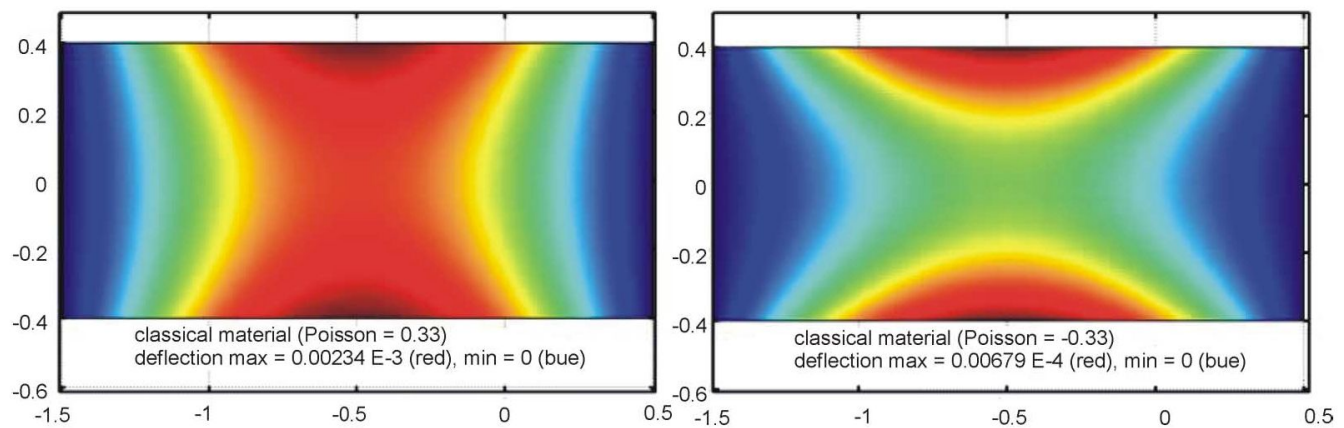

Fig. 10. First-order shear deformation (Mindlin) plate in bending: deflections with classical and auxetic material

conditions at the left and right edges and a uniform distribution of bending moments with opposite side at the upper and lower sides respectively. The discrtetization of the problem and the boundary conditions are shown in Fig. 9. The shape of the deformation (deflections of the plate) is indeed influenced, at it can be shown from Fig. 10. Let us mention that the considered problem is a very simple approximation of the plate in bending with an actuator acting in the vertical direction of the plate. 


\section{SUMMARY AND CONCLUSIONS}

Based on the shear deformation beam theory and Hamilton's principle, an efficient and more accurate finite element model for the shape control of sandwich composite beams with classical and auxetic material core and distributed piezoelectric actuators is developed. Related numerical results which demonstrate the effect of the auxetic material on static stress and displacement distributions are also presented. Within the theory of elasticity further investigation can demonstrate interface effects in materials with different Poisson's ratio. Furthermore the results of various beam theories, especially concerning the shear stress distribution and the transverse curvature, can be compared with the ones of three-dimensional simulations. Extensions in dynamic problems and problems of nonlinear mechanics, like strength and crack resistance, are beyond the scope of this paper. In particular, we would like to mention here the works on wave propagation in structures with auxetic core, see among others [18, 19], and the possible beneficial influence of the auxetic core on active control of smart beams and structures (cf. our previous work with classical materials published in [20, 21]). Relevant results will be published in the future elsewhere.

\section{References}

[1] H. Janocha (Ed.), Adaptronics and Smart Structures, Springer-Verlag Berlin (1999).

[2] P. Gaudenzi, F. Enrico, V. Koumousis, and C. Gantes, Shape control of statically indeterminate lami nated beams with piezoelectric actuators. J. Intell. Mater. Syst. 9, 291-300 (1998).

[3] B. N. Agrawal and K E. Treanor, Shape control of statically indeterminate laminated beams with piezoelectric actuators. Smart Mater. Struct. 8, 729-740 (1999).

[4] C. Chee, L. Tong, and G. Steven, A buildup voltage (BVD) algorithm for shape control of smart plate structures. Engineering Structures 24, 5-11 (2000).

[5] H. Irschik, A review on static and dynamic shape control of structures using piezoelectric actuation. Computational Mechanics 26, 115-128 (2002).

[6] E. P. Hadjigeorgiou, G. Foutsitzi, and G. E. Stavroulakis, Shape control of beams with piezo electric actuators Proc. 6th Hellenic-European Conference on Computer Mathematics and its Applications 25-27 September Athens HERCMA 2003, E. A. Lipitakis (Editor), LEA Publishers, Vol. I, pp. 164-168 (2003).

[7] Z. Friedman and J. B. Kosmatka, An inproved two-node Timoshenko beam finite element. Comput. Struct. 47 473-481 (1993).

[8] J. N. Reddy, On locking-free shear deformable beam finite elements. Comput. Methods Appl. Mech. Engrg. 149 113-132 (1997).

[9] W. Yang, Z. M. Li, W. Shi, B. H. Xie, and M. B. Yang. On auxetic materials. J. Mater. Sci. 39, 3269-3279 (2004).

[10] T. Matsuoka, S. Yamamoto, and M. Takahara, Prediction of structures and mechanical properties of composites using genetic algorithm andfinite element method. J. Mater. Sci. 36, 27-33 (2001).

[11] K E. Evans and A. Alderson, Auxetic materials: Functional materials and structures from lateral thinking. Adv. Mater. 12, 617628 (2000).

[12] G. E. Stavroulakis, Auxetic behaviour: appearance and engineering applications. Physica Status Solidi B, Special Issue on Auxetics and Related Systems, Guest Editor: K. Wojciechowski 2004 (in press).

[13] C. R. Houck, J. F. Joines, and M. G. Kay, A genetic algorithm for function optimization: A Matlab implementation. NCSU-IE TR 95-09 1995.

[14] H. F. Tiersten, Linear Piezoelectric Plate Vibration, Plenum Press New York (1969).

[15] H. S. Tzou, Piezoelectric Shells, Kluwer Academic Publishers The Netherlands (1993). 
[16] C. R. Cowper, The shear coefficient in Timoshenko's beam theory. ASME J. Appl. Mech. 33, 335-340 (1966).

[17] R. S. Lakes, Design considerations for negative Poisson's ratio materials. J. Mech. Design-T. ASME 115, 696-700 (1993).

[18] M. Ruzzene, L. Mazzarella, P. Tsopelas, and F. Scarpa, Wave propagation in sandwich plates with periodic auxetic core. Journal of Intelligent Material Systems and Structures 13(9), 587-597 (2002).

[19] M. Ruzzene and F. Scarpa, Control of wave propagation in sandwich beams with auxetic core. Journal of Intelligent Material Systems and Structures, 14(7), 443-453 (2003).

[20] G. Futsitzi, D. Marinova, E. Hadjigeorgiou and G. Stavroulakis, Robust H-2 vibration control of beams with piezoelectric sensors and actuators. Proceedings of Physics and Control Conference St. Petersburg Russia, 20-22.08.2003, Vol. 1, pp. 157-162.

[21] G. Stavroulakis, G. Futsitzi, E. Hadjigeorgiou, D. Marinova, and C. Baniotoloulos, Design of smart beamsfor suppresion of wind induced vibrations. The 9th International Conference on Civil and Structural Engineering Computing, Egmond and Zee, The Netherlands 2003, Paper 114, B.H.V. Topping (Editor), Civil-Comp Press. 